\title{
In eigener Sache
}

Hautarzt $2021 \cdot 72: 371-372$

https://doi.org/10.1007/s00105-021-04813-5

Angenommen: 23. März 2021

๑) Springer Medizin Verlag GmbH, ein Teil von Springer Nature 2021

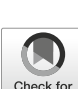

Check for
updates

Julie Kind' $\cdot$ Michael Jünger ${ }^{2}$ Alexander Kapp ${ }^{3} \cdot$ Roland Kaufmann ${ }^{4}$. Jean Krutmann ${ }^{5}$. Thomas Ruzicka ${ }^{6}$ für die Schriftleitung Der Hautarzt

${ }^{1}$ Redaktion Fachzeitschriften Medizin, Springer Medizin Verlag GmbH, Heidelberg, Deutschland

${ }^{2}$ Klinik und Poliklinik für Hautkrankheiten, Universitätsmedizin Greifswald, Greifswald, Deutschland

${ }^{3}$ Klinik für Dermatologie, Allergologie und Venerologie, Medizinische Hochschule Hannover, Hannover, Deutschland

${ }^{4}$ Klinik für Dermatologie, Venerologie und Allergologie, Universitätsklinik Frankfurt, Frankfurt am Main, Deutschland

${ }^{5}$ IUF - Leibniz-Institut für Umweltmedizinische Forschung, Düsseldorf, Deutschland

${ }^{6}$ Klinik für Dermatologie und Allergologie, Ludwig-Maximilians-Universität München, München, Deutschland

\section{Herrn Professor Hans F. Merk zum Dank}

\section{Schriftleitung und Verlag verabschieden den langjährigen Schriftleiter der Zeitschrift Der Hautarzt}

Nach 19 Jahren als Schriftleiter scheidet Herr Prof. Dr. Hans. F. Merk (• Abb. 1) zum 01.05.2021 aus dem Herausgebergremium der Zeitschrift Der Hautarzt aus.

Hans F. Merk, in Wuppertal geboren, absolvierte nach dem pharmazeutischen Vorexamen das Studium der Humanmedizin in Düsseldorf. Im Jahr 1981 wurde ihm für die Dissertation der Homburg-Preis des Regensburger Kollegiums für Ärztliche Fortbildung verliehen. Hans Merk war viele Jahre an der Hautklinik der Universität Köln tätig, u.a. als Leiter der Allergie-Abteilung. Im Jahr 1994 wurde er zum Universitätsprofessor für Dermatologie und Venerologie ernannt und stand der Hautklinik (Klinik für Dermatologie und Allergologie) der Medizinischen Fakultät der RWTH Aachen bis zu seiner Emeritierung im Jahr 2015 als Direktor vor.

Der Springer-Verlag schätzte sich 2002 sehr glücklich, mit Hans Merk einen Experten auf den Gebieten Allergologie, Dermatopharmakologie und -toxikologie für das Schriftleiterteam $\mathrm{zu}$ gewinnen. Mit seinem bemerkenswerten Erfahrungsschatz, seinem tiefen Interesse und seiner Freude an der Wis- senschaft setzte er mit durchschnittlich einem Leitthemenheft pro Jahr v. a. allergologische, dermatopharmakologische und berufsdermatologische Schwerpunkte - entsprechend seinem Zitat im Jubiläums-Editorial im März 2003 [1]:

In den kommenden Jahren sind für Dermatologie und Allergologie viele wesentliche Änderungen bezüglich neuer wissenschaftlicher Erkenntnisse, diagnostischer und therapeutischer Möglichkeiten sowie politischer Rahmenbedingungen zu erwarten. Durch die Bearbeitung vielfältiger Schwerpunktthemen in "Der Hautarzt“ wird gerade dieser Aspekt konzeptionell berücksichtigt.

Dank seiner zahlreichen Funktionen in unterschiedlichen Kommissionen und seines großen, (inter)nationalen Netzwerkes fiel es Hans Merk nie schwer, gefragte Autoren für die Leitthemenhefte zu gewinnen. Auch nach seiner Emeritierung brachte er sich mit Themenvorschlägen und deren Umsetzung weiterhin aktiv in die Gestaltung der Zeitschrift ein. Dass Ruhestand für ihn kein Rückzug aus der dermatologischen Wissenschaft bedeutete, zeigte sich auch darin, dass er weiterhin regelmäßig nach
Artikeln aus verschiedenen SpringerZeitschriften und -Büchern fragte, die er für Gutachten oder Fragestellungen in seinen noch vielfältigen Gremien und Ämtern brauchte. So ist Hans Merk u. a. noch Mitglied der Leopoldina, der Kosmetik-Kommission des Bundesamts für Risikobewertung (BfR) oder der Arbeitsgruppe „Haut und Allergie“ der MAK(Maximale Arbeitsplatzkonzentrationen)-Kommission der Deutschen Forschungsgemeinschaft (DFG).

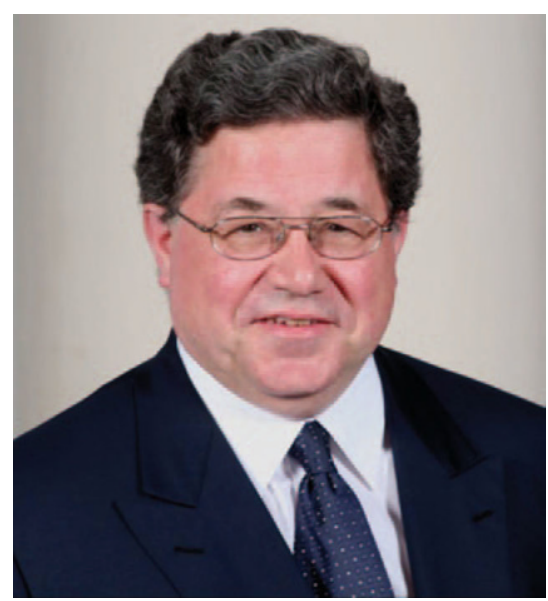

Abb. $1 \Delta$ Prof. Dr. med. Hans F. Merk 
Bei im Board $\mathrm{zu}$ treffenden Entscheidungen setzte sich Hans Merk mit einer ruhigen, zurückhaltenden Nachdrücklichkeit für die ihm wichtigen Themen ein. Das erste Thema des OneMinute-Wonder-Konzepts gefiel ihm sehr, denn es befasste sich mit einem Zytochrom(CYP)-Thema, eines seiner wissenschaftlichen Steckenpferde.

Lieber Herr Prof. Merk, Herausgebergremium und Verlag danken Ihnen sehr herzlich für die vertrauensvolle, bereichernde und erfolgreiche Zusammenarbeit. Es war uns eine große Freude und Ehre, dass Sie die Zeitschrift fast 2 Jahrzehnte als Schriftleiter begleitet und weiterentwickelt haben.

Mit den besten Wünschen

Schriftleitung und Herausgeber Der Hautarzt

Redaktion Der Hautarzt

\section{Korrespondenzadresse}

\section{Dr. med. Julie Kind}

Redaktion Fachzeitschriften Medizin, Springer Medizin Verlag $\mathrm{GmbH}$

Tiergartenstr. 17, 69121 Heidelberg,

Deutschland

julie.kind@springer.com

Interessenkonflikt. J. Kind, M. Jünger, A. Kapp, R. Kaufmann, J. Krutmann und T. Ruzicka geben an, dass kein Interessenkonflikt besteht.

\section{Literatur}

1. Der Hautarzt im 60. Jahrgang. Die Schriftleitung gratuliert zum Jubiläum (2009). Hautarzt 60:264-266. https://doi.org/10.1007/s00105009-1763-8

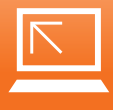

\section{Geben Sie uns Feedback!}

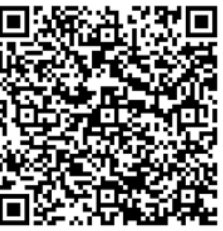

Liebe Leserinnen und Leser,

seit Jahresbeginn können Sie sich in jeder Hautarzt-Ausgabe auf ein One Minute Wonder freuen.

Folgende Themen werden bzw. wurden in der ersten Jahreshälfte 2021 kurz und

knapp zusammengefasst und visualisiert:

1/2021: Medikamentenwechselwirkungen. 1: Antimykotika und Phenprocoumon

2/2021: Photodynamische Therapie (PDT). Trends und neue Entwicklungen

3/2021: Medikamentenwechselwirkungen. 2: Orale Retinoide und Lipidsenker

4/2021: Fillerinjektion mit Hyaluronsäure. Was tun bei Gefäßverschluss?

5/2021: Vorsorge des Analkarzinoms

6/2021: Medikamentenwechselwirkungen. 3: QT-Zeit-Verlängerung

Gefallen Ihnen die Themenauswahl und die Umsetzung?

Wir sind neugierig auf Ihre Anregungen und Rückmeldungen zu dieser Reihe.

Wir freuen uns über Ihr Feedback!

Redaktion Der Hautarzt (julie.kind@springer.com) 